\title{
Opportunities for using biologically active substances Rhodiola rosea $L$. in the production of functional food with consideration for antimicrobial activity
}

\author{
Alexandra Zaushintsena ${ }^{1}$, Olga Babich $^{2}$, Evgeny Bryukhachev $^{1}{ }^{1}$ Irina Milenteva ${ }^{1}$, Anton Starcev ${ }^{1}$ \\ 1 Research Institute of Biotechnology, Kemerovo State University, Krasnaya Street 6, 650043, \\ Kemerovo, Russia \\ ${ }^{2}$ Institute of living systems Baltic Federal University Immanuel Kant, 236041, Kaliningrad, Russia
}

\begin{abstract}
Biologically active substances extricated by extraction of water and $40 \%$ ethyl alcohol from rootstalks of Rhodiola rosea L.: gallic acid, forms of rosovin and salidroside have been studied. Thin-layer chromatography (TLC) and high-performance liquid chromatography (HPLC) were used during the research. Determination of antimicrobial activity was performed by agar diffusion method (OFC.1.2.4.0010.15) [8]. The method is based on the evaluation of inhibition of the growth zone of testing cultures of microorganisms by certain concentrations of biologically active substances (BAS). String formed bacteria (8 strains), cocci bacteria (3) and fungal (4) microorganisms, bifidobacterium (10 strains) were used as testing cultures. Various responses of microorganisms to exposure by biologically active substances have been determined. Gallic acid and salidroside in the aqueous phase have the maximum inhibitory property to rod bacteria and coccoid microorganisms. Post-antibiotic effect is shown in rozovin. Salidroside in the butanol phase is more neutral to most microorganisms, with the exclusion of: Acinetobacter baumannii, Pseudomonas aeruginosa, Bacillus cereus and Staphylococcus aureus. The lack of a clearly expressed reaction of BAS to bifidobacterium, most probably, gives the possibility of their use in the development of functional products of a new generation. However, first of all, it is required to organize a special experiment on the structure of the introduction of the studied components into the lactoserum. Next, determine the content of BAS in the derived product, the reaction of bifidobacterium admitted for use in biotechnology, and a standard set of microbiological strains. Gallic acid is suggested to be introduced in applied studies to explore as a target immunomodulator of resistance to the listed microflora and to develop an effective form of a new product.
\end{abstract}

\section{Introduction}

\footnotetext{
* Corresponding author: alexaz58@yandex.ru
} 
Food technology improvement is topical and is related to many factors: the number and health of the population, climatic features of habitat, working conditions, and others. In accordance with modern estimates of researchers, the population continues to increase, and the volume of food resources can almost increase by no more than 1.3-1.5 times. There are two alternative problems: malnutrition and obesity. About 800 million people are malnourished: in developed countries, about $5 \%$ of the total population, in developing countries $-12.9 \%$, in Africa $-18.5 \%$, in low-income countries-26.1\% [1]. The diet in most cases is depleted of trace elements, vitamins, but enriched with fat. This results in serious physiological changes in the gastrointestinal tract and obesity development. Thereby, there is a lesion of blood vessels, bones, severe diseases development and associated diseases of the cardiovascular system, gastrointestinal tract, liver, including cancer.

It has been established that intestinal microbiocenosis is a highly sensitive, indicator system. It instantly reacts with quantitative and qualitative leapes to homeostasis changes [2]. Accordingly, it is recommended to use dairy products or functional products based on them more often. The nutritional value of milk is determined by the following useful composition: protein, fat, minerals, lactose, vitamins, enzymes, hormones, pigments, immune bodies, phosphotides, sterols, non-protein nitrogenous substances. Whey proteins, whose biological value in relation to the $\mathrm{FAO} / \mathrm{WHO}$ scale is $112 \%$, are not sufficiently implemented in food biotechnologies, while the protein deficit is at the level of 8-11 million tons $[3,4]$.

Serum is increasingly used as the main component in biotechnologies with the presence of herbal extracts that enrich the product with biologically active substances having antimicrobial, adaptogenic, immunomodulatory, oncogenic properties [5-7]. Herewith, attention should be paid to the resistance of probiotic and non-pathogenic microflora of serum and gastrointestinal tract towards biologically active substances of herbs.

Objective: to investigate the antimicrobial activity of biologically active substances of Rhodiola rosea L.)

\section{Materials and Methods}

Biologically active substances extricated by extraction of water and $40 \%$ ethyl alcohol from rootstalks of (Rhodiola rosea L.) the research object. Among them there were congeners of phenylpropanoids (rosavin from the water phase and rosavin from the ethanol phase) and phenol compounds (gallic acid, salidroside, salidroside of the butanol phase). Thin-layer chromatography (TLC) and high-performance liquid chromatography (HPLC) were used during the study). For extraction, air-dry plant raw materials were subjected to exhaustive extraction with $70 \%$ ethyl alcohol, the resulting water-alcohol extracts were evaporated under vacuum to a thick residue and then exposed to chromatographic separation. The chromatographic column (silica gel L 40/100) was eluted with chloroform and a mixture of chloroform - ethyl alcohol in different proportions. The separation of substances was controlled using TLC analysis on plates "Sorbfil PTSX-AF-a-UV" in the systems chloroform-ethanol (9:1), chloroform - methanol - water (26:14:3), and n-butanol - glacial acetic acid - water (4:1:2). Antimicrobial activity was identified by agar diffusion method (OFS.1.2.4.0010.15) [8]. The method is built upon the assessment of inhibition of the growth zone of testing cultures of microorganisms by certain densities of biologically active substances (BAS). Rod-shaped bacteria were used as testing cultures: Acinetobacter baumannii, Pseudomonas aeruginosa, Klebsiella pneumoniae, Serracia marcescens, Escherichia coli, Morganella morganii, Hafnia alvei, Bacillus cereus. We also identified the coccoid microflora reaction (Staphylococcus aureus, Staphylococcus epidermidis, Enterococcus faecium) and four strains of fungal flora from Candida (Candida non albicans No 136, 138, 142, 144). Bifidobacterium dentium (No84, No88), Bifidobacterium breve 
(No21,58,87), Bifidobacterium bifidum (No20,68), Bifidobacterium adolescentis (No85,88) were tested among bifidobacterium.

The tested cultures were preliminary grown on mown meat infusion agar (MIA) (FBUN GNTS PMB, Obolensk) for 24 hours at a temperature of $37{ }^{\circ} \mathrm{C}$. Next, a suspension of microorganisms was prepared in a sterile isotonic solution of $\mathrm{NaCl}$ and adjusted to a turbidity standard of 0.5 units by Mcfarland, which was consistent with a concentration of $1.5 \times 108$ $\mathrm{CFU} / \mathrm{ml}$. Inoculum was used for 15 minutes after preparationю It was applied with a pipette to the surface of a Petri dish with a Muller-Hinton medium (FBUN GNTS PMB, Obolensk) in a volume of 1-2 $\mathrm{ml}$. We equidistributed it on the surface with a sterile spatula. The slightly opened cups were dried at room temperature for 10-15 minutes. After that, extracts of biologically active substances from the callus of the common skullcap were applied with a sterile pipette. All microorganisms were incubated at $37^{\circ} \mathrm{C}$ for 24 hours. The result was assessed according to the following criteria: "-" - growth of the testing culture (without antibacterial effect); "+" - no growth (the antibacterial effect is pronounced); " \pm " - growth inhibition (bacteriostatic action).

\section{Results and Discussion}

Unique features of Rhodiola rosea proven by many researchers. Secondary metabolites modulates the phagocytic activity of white blood cells, lymphocyte proliferation and antibody production. They are widely used in medicine. Salidroside can be recommended for patients with bladder cancer [9]. A positive response to the depressive state of people has been revealed by affecting various components of the neuroendocrine, neurotransmitter, receptor and molecular networks related to a possible beneficial effect on mood [10].

Functional drinks and other products can have a positive effect on the central nervous system, activate mental activity and physical performance [11]. It is vital to know how active Rhodiola rosea extracts can be to non-pathogenic and conditionally pathogenic microflora of the gastrointestinal tract. It has been established that the main part of the microbial landscape of the colon of healthy people forms 1520 associations of dominant anaerobic, aerobic and facultative anaerobic types of bacteria: bifidobacterium, eubacterium, fusobacterium, clostridium, lactobacillus, bacilli, staphylococci, streptococci, enterococci, etc. [2].

Antimicrobial assessment of the studied extracts from the rhizome of Rhodiola rosea L. in relation to rod-shaped forms. It demonstrates a high level of inhibition of all testIng cultures during diffuse treatment of the crop using gallic acid [Fig. 1.]. Rosavin and salidroside completely inhibit Pseudomonas aeruginosa and B. cereus.

Table 1. Antimicrobial activity of BAS Rhodiola rosea

\begin{tabular}{|l|c|c|c|c|c|}
\hline \multirow{2}{*}{ Microorganisms } & \multicolumn{5}{|c|}{ Biologically active substances } \\
\cline { 2 - 6 } & gallic acid & rosavin & rosavin * & salidroside & salidroside ** \\
\hline $\begin{array}{l}\text { Acinetobacter } \\
\text { baumannii }\end{array}$ & + & \pm & \pm & \pm & \pm \\
\hline $\begin{array}{l}\text { Pseudomonas } \\
\text { aeruginosa }\end{array}$ & + & + & + & + & + \\
\hline $\begin{array}{l}\text { Klebsiella } \\
\text { pneumoniae }\end{array}$ & + & \pm & - & - & - \\
\hline $\begin{array}{l}\text { Serracia } \\
\text { marcescens }\end{array}$ & + & - & \pm & - & - \\
\hline $\begin{array}{l}\text { Escherichia coli } \\
\text { lac+ }\end{array}$ & + & \pm & - & + & - \\
\hline $\begin{array}{l}\text { Morganella } \\
\text { morganii }\end{array}$ & + & - & - & + & - \\
\hline
\end{tabular}




\begin{tabular}{|l|l|l|l|l|l|}
\hline Hafnia alvei & + & - & - & - & - \\
\hline Bacillus cereus & + & + & + & + & + \\
\hline
\end{tabular}

Note: rosavin* - water phase; salidroside**- butanol phase; " - "- growth of the test culture (no antibacterial effect); " + "- no growth (the antibacterial effect is pronounced); " \pm " - growth inhibition (bacteriostatic action).

The existence of rozovin in a functional product can restrain the growth of: Acinetobacter baumannii, Klebsiella pneumoniae Escherichia coli lac+, Serracia marcescens, and salidroside, - Acinetobacter baumannii. It was determined that all the studied biologically active substances have antimicrobial activity in relation to Staphylococcus aureus, and gallic acid also to Enterococcus faecalis [Fig. 2.]. There is no antifungicidal effect towards the flora of fungal origin.

Table 2. Antimicrobial activity of BAS

\begin{tabular}{|c|c|c|c|c|c|}
\hline \multirow[b]{2}{*}{ Microorganisms } & \multicolumn{5}{|c|}{ Biologically active substances of Rhodiola rosea } \\
\hline & gallic acid & rosavin & rosavin * & salidroside & $\begin{array}{c}\text { salidro } \\
\text { side } \\
* *\end{array}$ \\
\hline \multicolumn{6}{|c|}{ towards coccoid microflora } \\
\hline Staphylococcus aureus & + & + & + & + & + \\
\hline Enterococcus faecalis & + & - & - & - & - \\
\hline Enterococcus faecium & - & - & - & - & - \\
\hline \multicolumn{6}{|c|}{ towards Candida fungi } \\
\hline Candida non-albicans № 130 & - & - & - & - & - \\
\hline Candida non-albicans № 131 & - & - & - & - & - \\
\hline Candida non-albicans № 136 & - & + & - & - & - \\
\hline Candida non-albicans № 138 & - & - & - & - & - \\
\hline Candida non-albicans № 142 & - & - & - & - & - \\
\hline Candida non-albicans № 144 & - & - & - & - & - \\
\hline
\end{tabular}

Notes: "-" - test culture growth (no antifungal effect); "+" - lack of growth (antifungal effect is pronounced); “ \pm " - inhibition of growth (fungistatic action).

Biotechnologists seek two alternative goals: 1 - to increase the nutritional and functional value of a new functional product; 2.to maintain the optimal homeostatic balance of the gastrointestinal microbocenoses. Oftentimes, lactoserum is used for obtaining drinks for therapeutic and prophylactic purposes. It is saturated with probiotics based on bifidobacterium. BAS should be assessed based on its effect on bifidobacterium [3]. This evaluation phase showed the bacteriostatic action of ruzavin on Bifidobacterium dentium (No 88).

Table 3. Antibacterial activity of BAS of Rhodiola rosea (Rhodiola rosea L.) in respect to bifidobacteria

\begin{tabular}{|l|c|c|c|c|c|}
\hline \multirow{2}{*}{ Microorganisms } & \multicolumn{5}{|c|}{ Biologically active substances } \\
\cline { 2 - 6 } & gallic acid & rosavin & rosavin * & salidroside & salidroside ** \\
\hline $\begin{array}{l}\text { Bifidobacterium } \\
\text { dentium (№88) }\end{array}$ & - & \pm & - & - & - \\
\hline $\begin{array}{l}\text { Bifidobacterium } \\
\text { dentium (№84) }\end{array}$ & - & - & - & - & - \\
\hline Bifidobacterium & & & & & - \\
\hline
\end{tabular}




\begin{tabular}{|l|c|c|c|c|c|}
\hline breve (№58) & - & - & - & - & - \\
\hline $\begin{array}{l}\text { Bifidobacterium } \\
\text { breve (№21) }\end{array}$ & - & - & - & - & - \\
\hline $\begin{array}{l}\text { Bifidobacterium } \\
\text { breve (№87) }\end{array}$ & - & - & - & - & - \\
\hline $\begin{array}{l}\text { Bifidobacterium } \\
\text { bifidum (№68) }\end{array}$ & - & - & - & - & - \\
\hline $\begin{array}{l}\text { Bifidobacterium } \\
\text { bifidum (№20) }\end{array}$ & - & - & - & - & - \\
\hline $\begin{array}{l}\text { Bifidobacterium } \\
\text { bifidum } \\
\text { (npoбuomuческuü) }\end{array}$ & - & - & - & - & - \\
\hline $\begin{array}{l}\text { Bifidobacterium } \\
\text { adolescentis (№85) }\end{array}$ & - & - & - & - & - \\
\hline $\begin{array}{l}\text { Bifidobacterium } \\
\text { adolescentis (№88) }\end{array}$ & - & - & - & - & - \\
\hline
\end{tabular}

Note: rosavin* - water phase; salidroside**- butanol phase; " - "- growth of the test culture (no antibacterial effect); " + "- no growth (the antibacterial effect is pronounced); " \pm " - growth inhibition (bacteriostatic action).

\section{Conclusion}

As a result of the research, a different reaction of microorganisms to the effect of biologically active substances Rhodiola rosea L. was found. Gallic acid and salidroside in the aqueous phase have the maximum inhibitory ability to rod-shaped forms and coccoid microorganisms. Bacteriostatic effect is pronounced in rozavin. Salidroside in the butanol phase is more neutral to most microorganisms, with the exclusion of: Acinetobacter baumannii, Pseudomonas aeruginosa, Bacillus cereus and Staphylococcus aureus.

The absence of a well-defined reaction of BAS to bifidobacterium, likely, gives the possibility of their use in the development of functional products of a new generation. But first of all, it is required to organize a special experiment on the structure of the insertion of the studied components into the lactoserum. Next, determine the content of BAS in the resulting product, the reaction of bifidobacterium is approved for use in biotechnology, and a common set of microbiological strains.

Gallic acid is suggested to be used in applied researches on the study of resistance to the listed microflora as being an immunomodulator and for the development of an effective form of a new product.

\section{Acknowledgements}

The study was carried out within the framework of Agreement No. 075-02-2018-223 as of November 26, 2018 "Obtaining biologically active substances of endemic herbs to Siberia using cultures of cells and organs of higher plants" (unique project ID RFMEFI57718X0285).

\section{References}

1. A. Yu. Prosekov, Food safety: the challenge of modernity, Geoforum, 91, 73-77 (2018) DOI: https://doi.org/10.1016/j.geoforum.2018.02.030.

2. Ya.S. Tsimmerman, Klinicheskaya meditsina, 1, 4-11 (2013)

3. A.G. Khramtsov, Food Industry, 3, 23-29 (2017) 
4. A.G. Khramtsov, Food Industry, 1, 4-11 (2017)

5. C. Calcabrini, et al., Arch. Dermatol Res, 3, 191-200 (2010)

6. G.X. Mao, W.M. Xing, X.L. Wen, Int. J. Cosmet. Sci., 37 (3), 321-328 (2015)

7. K. Pulok Mukherjee, et al., Phytomedicine, 19, 64-73 (2011)

8. State pharmacopoeia of the USSR: General methods of analysis. Drug raw materials. Issue 2; 11-ed. Addition, 210 (Publisher: Medicine, 1990)

9. Li Yonghong, Victor Pham, Michelle Bui1, Liankun Song, Chunli Wu, Arman Walia, Edward Uchio, Feng Smith-Liu, Xiaolin Zi, Curr Pharmacol Rep, 3, 384-395 (2017) DOI 10.1007/s40495-017-0106-1.

10. Jay D. Amsterdam, Alexander G. Panossian, Phytomedicine, 23, 770 -783 (2016)

11. V. A. Utkin, Statistical technologies in medical researches, 214 (Pyatigorsk,2002) 